\title{
Estimación del Pronóstico Terapéutico y Análisis del Tratamiento en un Servicio Universitario de Psicología
}

\author{
Estimation of the Therapeutic Prognosis and Treatment Analysis in a College \\ Psychology Service
}

Federico Hervías Ortega ${ }^{1}$, Esperanza Marchena Consejero² y Inmaculada Menacho Jiménez ${ }^{3}$

\begin{abstract}
Resumen
La prevalencia de problemas psicológicos puede tener efectos negativos directos e indirectos en el desarrollo personal y académico de los estudiantes universitarios. A partir de una muestra de 43 participantes atendidos en el Servicio de Atención Psicológica de la Universidad de Cádiz se evalúa el impacto de la intervención realizada sobre sus niveles de salud mental a través del cuestionario Clinical Outcomes in Routine Evaluation-Outcome Measure (CORE-OM), y sus repercusiones en diferentes aspectos universitarios como la tasa de rendimiento o las calificaciones académicas. Se ha utilizado un modelo de cadenas de Markov con objeto de estudiar el comportamiento del sistema que represente el pronóstico de la evolución de los usuarios atendidos. Tomando como estados seis niveles de gravedad (de saludable a severo) que ofrece el CORE-OM se establecen las transiciones del alumnado entre los distintos estatus en un ciclo de 6 meses.
\end{abstract}

Palabras clave: servicio universitario de psicología, salud mental, pronóstico, Cadenas de Markov

\begin{abstract}
The prevalence of psychological problems may have directly and indirectly negative impacts on the personal and academic development of college students. Having taken a sample of 43 participants who were assessed by the College Psychology Service of University of Cádiz (Spain), the impact of the intervention implemented over their levels of mental health was evaluated using the questionnaire Clinical Outcomes in Routine Evaluation-Outcome Measure (CORE-OM), as well as their influence on different university aspects such as the rate of performance or academic qualifications. A Markov chain model was created with the purpose of studying the behavior of the system which represents the prediction of the evolution of cared students. Taking into consideration the six severity levels (from healthy to severe) offered by CORE-OM, the student transition among different statuses in a 6-month cycle was established.
\end{abstract}

Keywords: college psychology service, mental health, prognosis, Markov chain model

\footnotetext{
${ }^{1}$ Doctor en Psicología. Profesor del Departamento de Psicología, en el área de Personalidad, Evaluación y Tratamiento Psicológico. Universidad de Cádiz, España. Departamento de Psicología. Facultad de Ciencias de la Educación. Campus Río San Pedro. 11510 Puerto Real. Cádiz, España. Tel.: 617222043. Correo: federico.hervias@uca.es

${ }^{2}$ Doctora en Psicología. Profesora del Departamento de Psicología, en el área de Personalidad, Evaluación y Tratamiento Psicológico. Universidad de Cádiz, España. Departamento de Psicología. Facultad de Ciencias de la Educación. Campus Río San Pedro. 11510 Puerto Real. Cádiz, España. Correo: esperanza.marchena@uca.es

3 Doctora en Psicología. Profesora del Departamento de Psicología, en el área de Psicología Evolutiva y de la Educación. Universidad de Cádiz, España. Departamento de Psicología. Facultad de Ciencias de la Educación. Campus Río San Pedro. 11510 Puerto Real. Cádiz, España. Correo: inmaculada.menacho@uca.es

Revista Iberoamericana de Diagnóstico y Evaluación - e Avaliação Psicológica. RIDEP · No54 · Vol.1 · 69-80 2020 ISSN: 1135-3848 print /2183-6051online
} 


\section{Introducción}

A lo largo de los últimos años han aparecido en el organigrama universitario los llamados Servicios para Estudiantes, participando de forma activa en la evolución de las instituciones de Educación Superior (Murray, McKenzie, Murray, \& Richelieu, 2015). Así, en la actualidad es más frecuente que las universidades se preocupen por garantizar el rendimiento de los estudiantes a través de actividades complementarias para el estudio, dirigidas por los servicios de apoyo a los estudiantes (Sursock, 2015). En esta línea, se ha constatado la existencia de perfiles cada vez más dispares entre el alumnado universitario, debido en parte a las diversas vías de acceso existentes a los estudios superiores y la creciente movilidad de estudiantes a nivel internacional, fenómeno que hace más necesario este tipo de servicios. De entre los servicios de atención al estudiante existentes se incluyen los servicios de orientación académica, alojamiento, instalaciones deportivas, información sobre oportunidades de estudio y desarrollo profesional, capacitación en idiomas, actividades sociales y culturales y asesoramiento psicológico. Actualmente, se constata un aumento de la presencia de servicios de asesoramiento psicológico (Sursock \& Smidt, 2010), pasando de un 55\% de instituciones europeas que cuentan con este tipo de servicios en 2007, al 66\% que existían en 2010 y un $81 \%$ en el 2015. Este aumento, también constatado en las universidades españolas (Saúl, López-González, \& Bermejo, 2009), pone de manifiesto la creciente visión integral del alumnado y confirma la importancia no sólo de los aspectos académicos en el desarrollo de los estudiantes universitarios.

Ante este panorama, la prevalencia de trastornos mentales en población universitaria ha sido examinada en muchas ocasiones, ya que los estudiantes suelen ser utilizados como una muestra de fácil acceso de cara a realizar investigaciones en el campo de la psicopatología y otras áreas (Chau \& Saravia, 2016; MorenoYaguana \& Sánchez-García, 2018).

Así, se encuentran estudios epidemiológicos relacionados con la psicopatología general, haciendo especial hincapié en los trastornos del estado de ánimo y los trastornos de ansiedad (Vázquez \& Blanco, 2008). Es bien conocido que el riesgo de padecer algún trastorno emocional se incrementa bajo situaciones de estrés, y los estudiantes suelen vivir periodos sometidos a mucha tensión. De esta manera se han encontrado altos niveles de ansiedad y depresión en el alumnado, así como la existencia de una incidencia considerable de trastornos adaptativos respecto a otros trastornos psicológicos (Micin \& Bagladi, 2011).

En varias investigaciones se ha comprobado cómo los niveles de ansiedad se disparan en la población estudiantil en determinados momentos críticos, tales como la adaptación al contexto universitario en el primer año, periodos de evaluación, carga superior de trabajo o el momento de terminar los estudios y decidir si continuar con la formación o ingresar en el mercado laboral (Agudelo, Casadiegos, \& Sánchez, 2008; Arco, Fernández, Heilborn, \& López, 2005), por lo que su impacto en la vida de los estudiantes se hace patente en numerosas ocasiones.

Respecto a los trastornos del estado de ánimo, los estudios de prevalencia ofrecen resultados muy dispares, situando la población con problemas de depresión entre el 8\% (Vázquez \& Blanco, 2008) y el 21\% (Arslan, Ayranci, Unsal, $\&$ Arslantas, 2009). Se ha estudiado el efecto de la depresión en el rendimiento académico (Sarokhani et al., 2013), encontrándose una estrecha relación entre estados depresivos y ciertos desajustes en la vida del estudiante, como bajos niveles de concentración, ausencia injustificada a las clases, considerar abandonar los estudios o el consumo de alcohol y otras drogas (Gutiérrez et al., 2010).

Por otro lado, las demandas propias de la universidad ha generado la aparición de problemas específicos asociados a la vida académica, con graves efectos en el rendimiento de los estudiantes, como la ansiedad a los exámenes, la fobia escolar, el choque cultural en el caso de los estudiantes internacionales o comportamientos problemáticos que suelen presentarse de forma generalizada, como la procrastinación o problemas de socialización.

Existes estudios acerca del efecto de un proceso terapéutico sobre el rendimiento académico en alumnos con dificultades, llegando a distintas conclusiones. Un ejemplo lo 
encontramos en Arco, López, Heilborn y Fernández (2005) donde a través de un programa conductual basado en terapia breve (complementado con elementos de la Terapia de Aceptación y Compromiso) se consigue mejorar aspectos como mantener un horario fijo de actividades y número de horas de estudio, asistencia regular a clase, regulación de los patrones de sueño o una disminución de la irritabilidad. En una revisión sobre estudios acerca de la relación entre resultados académicos y otras variables psicológicas (Richardson, Abraham, \& Bond, 2012) se encontraron variables no relacionadas con aspectos cognitivos que interaccionan con el rendimiento, como factores motivacionales o estrategias de autorregulación del aprendizaje, factores con los que se trabaja en los servicios universitarios de atención psicológica. La mejora de la autoestima también se ha relacionado con una mejora en el desempeño académico (Gębka, 2013; Pullmann \& Allik, 2008). Sin embargo también contamos con trabajos donde no se niega la influencia de variables psicológicas, pero que otorgan una mayor importancia a variables como las calificaciones en las etapas educativas previas (Olani, 2009) o las competencias cognitivas (Prat-Sala \& Redford, 2010) como determinantes del desempeño académico en la universidad. Hay estudios en donde incluso se identifican estudiantes académicamente exitosos que puntúan alto en escalas clínicas (Stringer, Crown, Lucas, \& Supramanium, 1977), rompiendo esa conexión entre rendimiento y salud mental.

$\mathrm{Si}$ bien la atención psicológica a nivel académico comienza a tener mayor visibilidad, se necesitan estudios contrastados que permitan valorar el beneficio que aporta al desempeño académico y bienestar personal de los estudiantes. La aplicación del modelo de cadenas de Markov facilitará conocer el pronóstico de la intervención realizada por los profesionales de los servicios universitarios de atención psicológica.

Los modelos Markov son estructuras analíticas que representan un estado en un conjunto de posibles estados mutuamente excluyentes y colectivamente exhaustivos relacionados entre sí por probabilidades de transición, y a través de los cuales una persona o una cohorte pueden transitar en un número definido de ciclos de igual valor. En situaciones en la que los parámetros de los procesos de decisión están afectados por cuestiones previas, o por variables externas de carácter cronológicamente dinámico, son de gran utilidad. Esta aplicación, es el punto de partida para diseñar un modelo de procesos que implican conductas o elecciones personales a lo largo de un espacio de tiempo. Este tipo de modelos permite realizar un análisis de predicción al tiempo que uno de estructura interna. Las matrices de transición se han revelado como instrumentos valiosos en el estudio de la conducta, porque respetan su propia dinámica sin reducirla a una visión discreta de un suceso que es esencialmente temporal; pero, además, permiten predecir, mediante cadenas probabilísticas, la transición de una fase a otra dentro de una secuencia, y anticipar el estado más probable en que un sujeto va a ser observado cuando tal proceso alcanza su estacionalidad o equilibrio. No obstante, las cadenas de Markov como cualquier procedimiento estadístico tienen sus limitaciones a la hora de interpretar sus resultados. Por ejemplo, las probabilidades de transición entre estados en un ciclo se asumen invariables en el tiempo, e idénticas para todas las personas, sin tener en cuenta la existencia de variables extrañas y/o contaminantes que afecten en su desarrollo.

En este estudio se establecerán las transiciones del alumnado entre distintos estados de salud (saludable, bajo, leve, moderado, moderado-severo y severo) en tres ciclos de 6,12 y 18 meses.

\section{Método}

\section{Participantes}

Han participado 43 estudiantes atendidos durante dos cursos académicos, y seleccionados a través de un muestreo de casos críticos. Todos ellos completaron el protocolo de intervención del Servicio de Atención Psicológica (SAP) de la Universidad de Cádiz (UCA), incluyendo las evaluaciones pretest y postest. La distribución por sexos reflejó un $44.2 \%$ de hombres y un $55.8 \%$ de mujeres. El rango de edad está comprendido entre los 18 y 56 años $(M=23.37 ; D T=6.96)$, aunque el mayor porcentaje de alumnos se sitúa en un intervalo entre los 18 y 23 años (76.7\%).

Un aspecto importante a resaltar es la necesidad de contar con un grupo control que 
permita dar mayor consistencia a los resultados, sin bien en ocasiones es posible cuantificar la eficacia en sí misma en términos probabilísticos sin la presencia de un grupo comparativo.

En cuanto al diagnóstico general de los usuarios del SAP, el $34.9 \%$ corresponden a problemas psicopedagógicos, seguido de trastornos de tipo psicopatológico según criterios DSM-IV relacionados con la ansiedad (23.3\%), trastornos de estado de ánimo (9.3\%), trastornos de la personalidad (7\%), trastornos de la conducta alimentaria (2.3\%), trastornos adaptativos $(2,3 \%)$, trastornos sexuales $(2.3 \%)$ y otros problemas que puedan ser objeto de atención clínica (18.6\%). Todo el proceso de acogida, evaluación, diagnóstico e intervención se lleva a cabo por los profesionales que trabajan o colaboran con el SAP, siendo todos ellos psicólogos titulados y con la acreditación sanitaria necesaria para llevar a cabo tareas asistenciales en materia de salud. Se contemplan aspectos comunes en la intervención realizada como el procedimiento de evaluaciónintervención-seguimiento, los instrumentos de acogida y evaluación, criterios diagnósticos utilizados, un número máximo de sesiones de tratamiento o un enfoque terapéutico basado en el modelo cognitivo-conductual. Los elementos diferenciadores pueden localizarse en el número definitivo de sesiones llevadas a cabo, la localización temporal de la acogida de los sujetos (comienzos del curso, a mitad de un semestre, al acercarse un periodo lectivo complicado, después de la época de exámenes, etc.) y el propio estilo personal del terapeuta, variable que ha demostrado ser muy relevante en relación al resultado de una intervención o al establecimiento de una correcta alianza terapéutica (Casari, Isón, \& Gómez, 2018).

\section{Instrumentos}

Clinical Outcomes in Routine Evaluation Outcome Measure (CORE-OM) de Chris Evans (1998). Instrumento de evaluación diseñado para medir el cambio terapéutico producido tras un proceso de intervención psicológica (Barkham et al., 2001). Consta de 34 ítems autoadministrados que evalúan el estado del sujeto en 4 dimensiones, algunas de ellas divididas a su vez en distintas áreas. Las subescalas son Bienestar Subjetivo (W); Problemas y Síntomas (P) relacionados con ansiedad, depresión, problemas físicos y trauma; Funcionamiento $(\mathrm{F})$ a nivel general y concerniente a relaciones cercanas y relaciones sociales; y Riesgo (R) de autolesiones y hacia otros, además de una escala general denominada Visibilidad (Visi). En el caso de una puntuación nula en la escala $\mathrm{R}$, se recomienda eliminarla de la valoración global, encontrándose la escala Visi-R Los ítems se puntúan de 0 a 4 y se refieren a la sintomatología experimentada durante los 7 días anteriores a la realización de la prueba. Cabe resaltar que la escala Bienestar Subjetivo sigue una puntuación inversa, de forma que puntuaciones elevadas en el instrumento indican problemas relacionados con el bienestar subjetivo. Las puntuaciones directas de CORE -OM pueden variar de 0 a 136, indicando mayor severidad con puntuaciones más elevadas, estableciéndose los siguientes niveles de gravedad: saludable (1-20), bajo (21-22), leve (34-50), moderado (51-67), moderado a severo (68-84) y severo (85-136). La consistencia interna es de un $\alpha=.94$ (Barkham et al., 2001), con un punto de corte entre población clínica y no clínica de 1.19 para los hombres y 1.29 para las mujeres en la escala Visibilidad. Sus propiedades psicométricas han demostrado niveles aceptables de consistencia interna y sensibilidad en las medidas obtenidas (Evans et al., 2002), y ha sido utilizado en numerosos contextos clínicos (Connell, et al., 2007; Palmieri, et al., 2009); y universitarios (Botella, 2008; Young, 2009). Se recomienda utilizar la herramienta antes y después del tratamiento, obteniendo así una medida pretest y postest, las cuáles se comparan para conseguir una estimación de los resultados alcanzados.

Ficha de acogida de los usuarios del SAP. Instrumento confeccionado ad hoc en el que se recoge información de los estudiantes que son atendidos en sesiones individuales. Dicho instrumento se completa por el usuario del SAP antes de la primera cita presencial, de manera que la información recogida sirva para preparar la primera entrevista personal.

\section{Procedimiento}

Se obtuvo el consentimiento informado de cada uno de los estudiantes para llevar a cabo el desarrollo de la investigación. Cada una de las pruebas se administró de manera individual, diferenciándose cuatro fases en este estudio: 
$1^{\text {a }}$ fase: Recepción de la demanda del estudiante: los estudiantes solicitan una atención individualizada con el SAP, completando la ficha de acogida y firmando el consentimiento informado.

$2^{a}$ fase: Evaluación Pretest: al comienzo de la intervención, durante el proceso de entrevista y evaluación, se administró el cuestionario COREOM.

$3^{\text {a }}$ fase: Proceso de asesoramiento $e$ intervención: a lo largo de un número de sesiones, que oscilan entre 1 y 10 , el estudiante se somete al proceso de asesoramiento e intervención individualizado mediante terapia cognitivo conductual, incluyendo activación conductual, reestructuración cognitiva, relajación aplicada y resolución de problemas, entre otras (Marchena et al, 2009). El proceso de actuación se basa en un modelo de intervención breve, donde a través de un número establecido de sesiones, se proponen los objetivos que se persiguen y se abordan de manera consecutiva. Dichos objetivos vienen definidos por el diagnóstico establecido y las expectativas de los usuarios atendidos. Después de cada sesión el profesional que atiende el caso debe completar una ficha de seguimiento donde se especifican los objetivos propuestos, tareas pendientes a realizar, el seguimiento de las sesiones o cualquier otro tipo de información que se considere relevante para la intervención. Se contemplan aspectos comunes en las intervenciones realizadas como el procedimiento de evaluación-tratamiento-seguimiento, los instrumentos de acogida y evaluación, criterios diagnósticos utilizados, un número máximo de sesiones de tratamiento o un enfoque terapéutico basado en el modelo cognitivo-conductual. Los elementos diferenciadores pueden localizarse en el diagnóstico establecido (que condiciona a su vez los objetivos terapéuticos), el número definitivo de sesiones llevadas a cabo, la localización temporal de la acogida de los sujetos (comienzos del curso, a mitad de un semestre, al acercarse un periodo lectivo complicado, después de la época de exámenes, etc.) y el propio estilo personal del terapeuta, variable que ha demostrado ser muy relevante en relación al resultado de una intervención o al establecimiento de una correcta alianza terapéutica (Casari, Isón, \& Gómez, 2018).

$4^{a}$ fase: Evaluación Postest: al término del proceso de intervención aplicando el cuestionario CORE-OM (aproximadamente 6 meses desde la recepción de la demanda).

\section{Análisis de Datos}

El tratamiento de los datos se ha llevado a cabo a través del sistema SPSS.20, incluyendo un análisis descriptivo de las puntuaciones obtenidas. Queriendo examinar el nivel de significación de las diferencias entre las puntuaciones pretest y postest, se realiza un contraste de hipótesis para muestras relacionadas bajo pruebas no paramétricas (prueba de Wilcoxon (Z) y de Signos). El pronóstico de la intervención se ha analizado generando un modelo de cadenas de Markov, con objeto de estudiar el comportamiento del sistema.

\section{Resultados}

Para conocer el impacto de la atención ofrecida desde el SAP, en primer lugar se realiza un análisis descriptivo de las escalas clínicas evaluadas en los momentos pretest y postest a partir de la puntuación directa. Tal y como se observa en la Tabla 1, en todas las subescalas del CORE-OM se produce un descenso en las puntuaciones medias desde la medida pretest (W: 9.33; P: 23.23; F: 18.67; R: 2.14) a la postest (W: 5.37; P: 11.88; F: 11.60; R: 1.07).

Tabla 1. Estadísticos descriptivos de las escalas clínicas pretest y postest del CORE-OM

\begin{tabular}{lcccccccc}
\hline & \multicolumn{4}{c}{ PRETEST } & \multicolumn{4}{c}{ POSTEST } \\
& Mín. & Máx. & Media & D.T. & Mín. & Máx. & Media & D.T. \\
\hline $\mathrm{W}$ & 1 & 16 & 9.33 & 3.235 & 0 & 13 & 5.37 & 3.295 \\
$\mathrm{P}$ & 7 & 43 & 23.23 & 8.890 & 0 & 37 & 11.88 & 8.686 \\
$\mathrm{~F}$ & 3 & 38 & 18.67 & 7.921 & 1 & 31 & 11.60 & 7.725 \\
$\mathrm{R}$ & 0 & 16 & 2.14 & 3.441 & 0 & 10 & 1.07 & 2.040 \\
Visi & 14 & 103 & 53.37 & 19.409 & 1 & 86 & 29.93 & 20.270 \\
Visi-R & 14 & 94 & 51.23 & 17.600 & 1 & 81 & 28.91 & 18.923 \\
\hline
\end{tabular}

W: Bienestar Subjetivo; P: Problemas y Síntomas; F: Funcionamiento; R: Riesgo; Visi: Visibilidad. 
Tabla 2. Prueba de Wilcoxon (Z) y de Signos. Estadísticos de contraste para muestras relacionadas.

\begin{tabular}{|c|c|c|c|c|c|c|c|c|c|}
\hline & \multirow{2}{*}{$\begin{array}{l}\text { Rangos }(-,+) \\
\text { Empates }(E)\end{array}$} & \multirow[b]{2}{*}{$\mathrm{N}$} & \multirow[b]{2}{*}{$\%$} & \multirow[b]{2}{*}{$\begin{array}{c}\text { Rango } \\
\text { promedio }\end{array}$} & \multirow[b]{2}{*}{$\begin{array}{l}\text { Suma de } \\
\text { rangos }\end{array}$} & \multicolumn{2}{|c|}{ Wilcoxon } & \multicolumn{2}{|c|}{ Signos } \\
\hline & & & & & & $\mathrm{Z}$ & $\begin{array}{l}\text { Sig.Asint } \\
\text { (bilat.) }\end{array}$ & $\mathrm{Z}$ & $\begin{array}{c}\text { Sig.Asint } \\
\text { (bilat.) }\end{array}$ \\
\hline \multirow{4}{*}{ W } & - & 40 & 93 & 22.40 & 896.00 & & & & \\
\hline & + & 2 & 4.6 & 3.50 & 7.00 & -5.573 & $.000 *$ & -5.709 & $.000 *$ \\
\hline & $\mathrm{E}$ & 1 & 2.4 & & & & & & \\
\hline & - & 40 & 93 & 22.33 & 893.00 & & & & \\
\hline \multirow[t]{3}{*}{$\mathrm{P}$} & + & 2 & 4.6 & 5.00 & 10.00 & -5.522 & $.000 *$ & -5.709 & $.000 *$ \\
\hline & $\mathrm{E}$ & 1 & 2.4 & & & & & & \\
\hline & - & 35 & 81.4 & 22.27 & 779.50 & & & & \\
\hline \multirow[t]{3}{*}{$\mathrm{F}$} & + & 6 & 14 & 13.58 & 81.50 & -4.526 & $.000 *$ & -4.373 & $.000 *$ \\
\hline & $\mathrm{E}$ & 2 & 4.6 & & & & & & \\
\hline & - & 18 & 41.9 & 11.61 & 209.00 & & & & \\
\hline \multirow[t]{3}{*}{$\mathrm{R}$} & + & 4 & 9.3 & 11.00 & 44.00 & -2.705 & $.007 *$ & & $* *$ \\
\hline & $\mathrm{E}$ & 21 & 48.8 & & & & & & \\
\hline & - & 39 & 90.7 & 23.79 & 928.00 & & & & \\
\hline \multirow[t]{3}{*}{ Visi } & + & 4 & 9.3 & 4.50 & 18.00 & -5.495 & $.000 *$ & -5.185 & $.000 *$ \\
\hline & $\mathrm{E}$ & 0 & 0 & & & & & & \\
\hline & - & 39 & 90.6 & 22.82 & 890.00 & & & & \\
\hline \multirow[t]{2}{*}{ Visi-R } & + & 3 & 7 & 4.33 & 13.00 & -5.484 & $.000 *$ & -5.401 & $.000 *$ \\
\hline & $\mathrm{E}$ & 1 & 2.4 & & & & & & \\
\hline
\end{tabular}

$* p<.05 \quad * *$ Sig. exacta - bilateral: .004 (se ha usado la distribución binomial)

Tabla 3. Matriz de probabilidad de transición de un estado a otro en función de los niveles de gravedad establecidos en el CORE-OM. Estadio 1

\begin{tabular}{|c|c|c|c|c|c|c|c|}
\hline & \multirow{2}{*}{ Estados } & \multicolumn{6}{|c|}{ Hacia } \\
\hline & & Saludable & Bajo & Leve & Moderado & Mod-Sev & Severo \\
\hline \multirow{6}{*}{$\begin{array}{l}\stackrel{0}{0} \\
\text { ஸे }\end{array}$} & Saludable & 1 & .00 & .00 & .00 & .00 & .00 \\
\hline & Bajo & 1 & .00 & .00 & .00 & .00 & .00 \\
\hline & Leve & .42 & .28 & .23 & .04 & .00 & .00 \\
\hline & Moderado & .30 & .10 & .40 & .20 & .00 & .00 \\
\hline & Mod-Sev & .25 & .25 & .00 & .25 & .00 & .25 \\
\hline & Severo & .25 & .00 & .25 & .00 & .50 & .00 \\
\hline
\end{tabular}

Parece claro que se ha reducido la experiencia subjetiva general de los síntomas asociados con las variables evaluadas en el alumnado atendido en el SAP tras recibir las pautas de asesoramiento (pretest V: $53.37 /$ postest V: 29.93 y pretest V-R: 51.23/ postest V-R: 28.91) (ver Tabla 2), pudiendo concluir que todas las variables comparadas difieren significativamente antes y después de la intervención.

Por otro lado, tomando como estados los seis niveles de gravedad que ofrece el cuestionario CORE-OM (saludable, bajo, leve, moderado, moderado-severo y severo), se establecen las transiciones del alumnado entre los distintos estatus en un ciclo de 6 meses. Al final de un ciclo, los estudiantes pueden permanecer en un estado o trasladarse a otro, con base a las probabilidades de transición que fueron previamente definidas a través del uso del tratamiento psicológico. Las flechas muestran la dirección de transición y su probabilidad. Cuando la flecha vuelve al mismo estado de salud, representa la probabilidad de permanencia en ese estado. En la estructura de modelización con intervención (Figura 1), cabe destacar las probabilidades de transición dominantes entre los estados Bajo vs. Saludable (1), Severo vs. Moderado-Severo (.50), Leve vs. Saludable (.42) y Moderado vs. Leve (.40). El estado Saludable es estable en el sentido de que los sujetos no tienen transiciones a otros estados. Es decir, de acuerdo con los resultados obtenidos, se observa que existe un $100 \%$ de probabilidad de que un usuario que se encuentre en un estado Bajo de salud pase a un estado Saludable en un periodo de 6 meses. Existe un $50 \%$ de probabilidad para que un estudiante en estado Severo pase a Moderado-Severo y $25 \%$ de probabilidad de que se pase a Leve o Saludable. Por último, un usuario en estado Leve tiene un $23 \%$ de permanecer en este estado, un $42 \%$ de que se pase a Saludable, $28 \%$ a Bajo y $4 \%$ a Moderado. Los datos de la Figura 1 se recogen en 
Tabla 4. Matriz de probabilidad de transición de un estado a otro en función de los niveles de gravedad establecidos en el CORE-OM. Estadios 2 y 3

\begin{tabular}{|c|c|c|c|c|c|c|c|}
\hline & \multirow{2}{*}{ Estados } & \multicolumn{6}{|c|}{ Hacia } \\
\hline & & Saludable & Bajo & Leve & Moderado & Mod-Sev & Severo \\
\hline \multirow{14}{*}{ 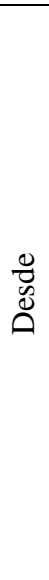 } & Estadio 2 & & & & & & \\
\hline & Saludable & 1 & .00 & .00 & .00 & .00 & .00 \\
\hline & Bajo & 1 & .00 & .00 & .00 & .00 & .00 \\
\hline & Leve & .83 & .07 & .08 & .02 & .00 & .00 \\
\hline & Moderado & .63 & .13 & .18 & .06 & .00 & .00 \\
\hline & Mod-Sev & .64 & .02 & .16 & .05 & .12 & .00 \\
\hline & Estadio 3 & & & & & & \\
\hline & Severo & .48 & .20 & .06 & .14 & .00 & .12 \\
\hline & Saludable & 1 & .00 & .00 & .00 & .00 & .00 \\
\hline & Bajo & 1 & .00 & .00 & .00 & .00 & .00 \\
\hline & Leve & .94 & .02 & .03 & .01 & .00 & .00 \\
\hline & Moderado & .86 & .06 & .07 & .02 & .00 & .00 \\
\hline & Mod-Sev & .78 & .08 & .06 & .05 & .00 & .03 \\
\hline & Severo & .78 & .03 & .10 & .03 & .06 & .00 \\
\hline
\end{tabular}

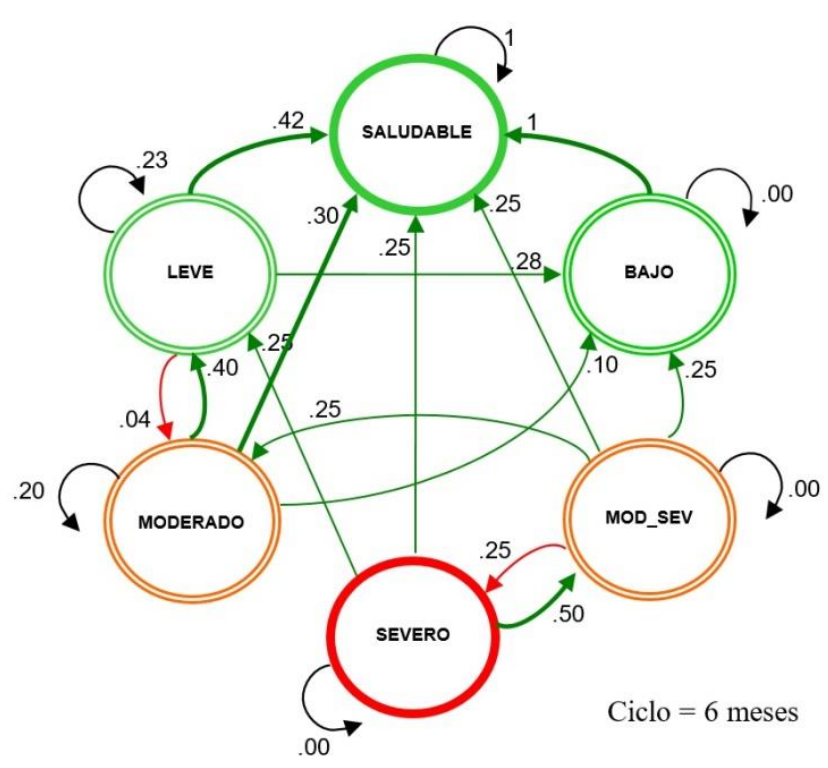

Figura 1. Representación gráfica del primer ciclo del Modelo de Markov - Estadio 1

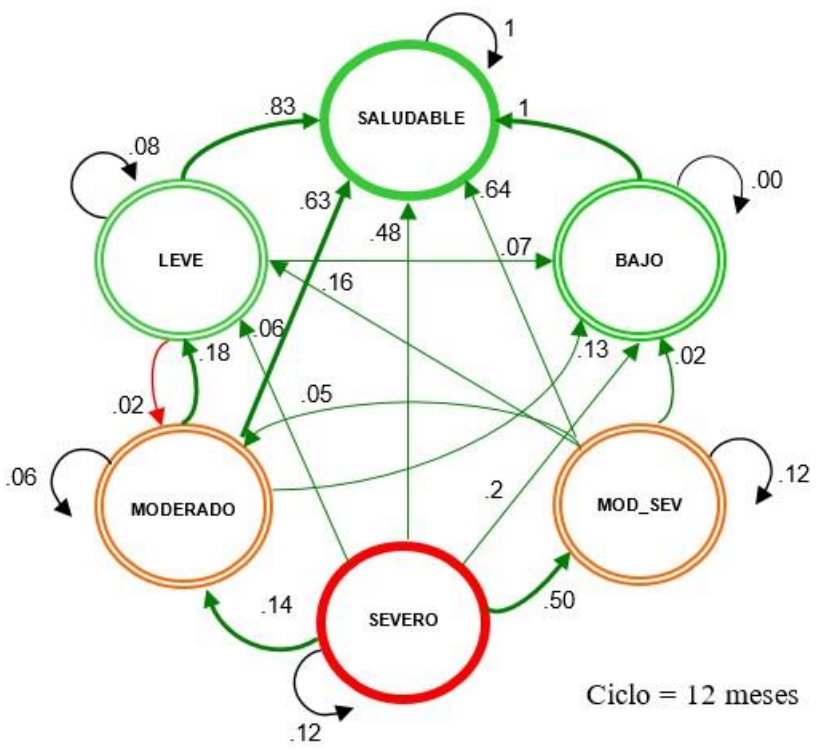

Figura 2. Representación gráfica del segundo ciclo del Modelo de Markov - Estadio 2

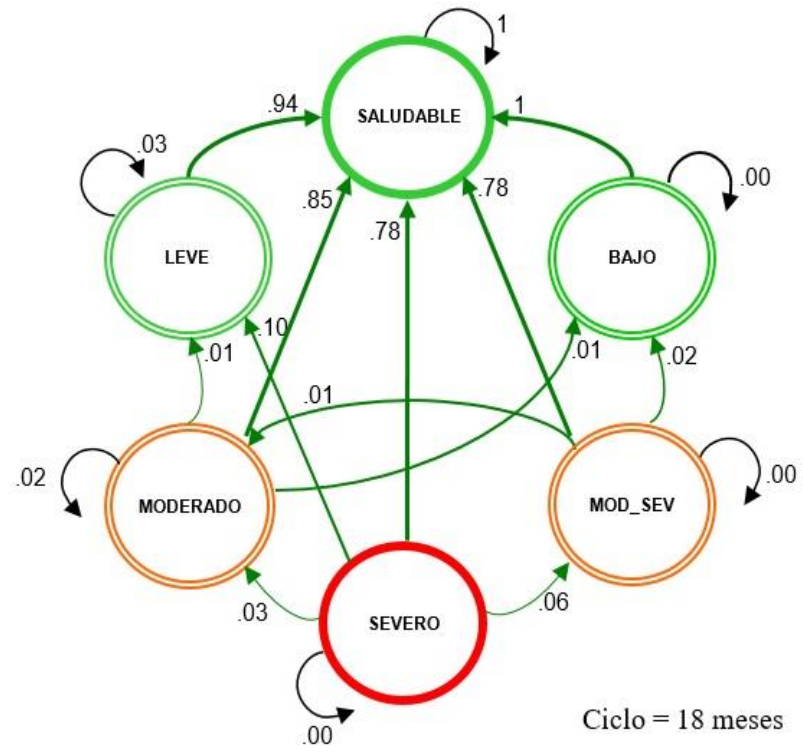

Figura 3. Representación gráfica del tercer ciclo del Modelo de Markov - Estadio 3

una matriz de probabilidades como la que se muestra en la Tabla 3. Cabe destacar que la suma de las probabilidades para cada fila en la matriz de transición P es siempre 1.

Para conocer la probabilidad de que el sistema se encuentre en un estado estable determinado se han realizado 3 transiciones. En la Tabla 4 se representa la matriz de probabilidades de los estadios 2 (a los 12 meses) y 3 (18 meses) (figuras 2 y 3 ). Se observa cómo, llegado este momento, el sistema alcanza un alto grado de estabilidad, saturando el modelo y produciéndose un efecto embudo hacia el estado saludable. 


\section{Discusión}

En el ámbito universitario existen muchas variables relacionadas con la salud mental que tienen una incidencia directa o indirecta sobre el desempeño académico y la adaptación de los estudiantes, como la gestión del estrés, la autorregulación emocional, los hábitos de vida, el consumo de alcohol y otras drogas o la calidad de las relaciones sociales que se establecen.

La puesta en marcha de programas de prevención dirigidos a jóvenes universitarios proporciona beneficios en cuanto a variables que inciden en el bienestar. El periodo universitario es un momento de vital importancia en el desarrollo de los estudiantes, en el que se conforma la personalidad del individuo y se produce el tránsito a la vida adulta, con la consecuente asunción de roles y obligaciones. Se trata por tanto un momento crucial para la fijación de hábitos saludables, minimizando la probabilidad de reproducir conductas de riesgo y sus consecuencias. Existen muchos ejemplos de programas de este tipo, abarcando aspectos relacionados con el consumo de alcohol (Bewick, Trusler, Mulhern, Barkham, \& Hill, 2008; Hallett, Maycock, Kypri, Howat, \& McManus, 2009), tabaco (Martín, Fernández, Ordóñez, Molina, \& Manuel, 2008), sustancias psicoactivas (Tirado, Aguaded, \& Marín, 2013) o adicciones comportamentales con programas dirigidos a reducir el abuso en la utilización de nuevas tecnologías (Quintero, Martín, \& Fuentes, 2012). También hay experiencias en la prevención de conductas sexuales de riesgo (Vladutiu, Martin, \& Macy, 2011) o relacionados con la violencia de género (Aguilar, Alonso, Melgar, \& Molina, 2009). De forma paralela, además de prevenir conductas de riesgo, se generan multitud de programas encaminados a fomentar buenos hábitos de vida en los estudiantes, promoviendo una adecuada alimentación (Dunne \& Somerset, 2004) o la práctica de una vida activa (Ortís et al., 2007).

Investigaciones realizadas ponen de manifiesto los múltiples beneficios ligados a la intervención psicológica en estudiantes (Lepp, Barkley \& Karpinski, 2014; Monti, Tonetti \& Ricci Bitti, 2015; Murray, McKenzie, Murray, \& Richelieu, 2015). Así, el asesoramiento e intervención psicológica deben ser parte integral del proceso de la Educación Superior, y de las estrategias para optimizar el estado de salud y bienestar del alumnado, especialmente para aquellos estudiantes que presentan trastornos o dificultades psicológicas.

A través del modelo, creado con las probabilidades de transición de un estado de salud a otro, se observa una tendencia generalizada de los estudiantes a confluir en el estado saludable tras la intervención en el SAP de la UCA. Este fenómeno se intensifica al generar un segundo modelo, en el que se crea un escenario ficticio donde el tiempo de la intervención se duplica, llegando a obtener un $89.7 \%$ de sujetos en el estado saludable.

De esta forma se confirma la hipótesis de que la intervención llevada a cabo con los estudiantes desde el SAP mejora su estado de salud mental, reduciendo considerablemente los valores en las escalas clínicas observadas. Estos resultados se unen a otras investigaciones que han demostrado la efectividad de los servicios de atención psicológica (Alcoba-González, 2000; Koutra, Katsiadrami, \& Diakogiannis, 2010; Minami et al., 2009; Raunic \& Xenos, 2008; Rosenthal \& Wilson, 2008). Estudios realizados utilizando la prueba CORE-OM (Connell, Barkham, \& MellorClark, 2007, 2008) también han detectado reducciones significativas en las puntuaciones ofrecidas por los usuarios de otros servicios de counselling universitarios, con un porcentaje que oscila entre el $67 \%$ y el $83 \%$ de casos en donde hay una reducción de las puntuaciones postest con respecto a las puntuaciones pretest. En este estudio, tomando como referencia la variable CORE-Visibilidad, el porcentaje de casos en donde se reducen las puntuaciones ha alcanzado a un $90.7 \%$ del total.

El alumnado universitario en riesgo de fracaso, bajo rendimiento o abandono necesita servicios de asesoramiento psicológico para prevenir o mitigar la sintomatología que se asocia a sus problemas psicológicos o psicopedagógicos (Mehr \& Daltry, 2016). El presente estudio proporciona argumentos para la defensa del uso de los servicios universitarios de atención psicológica como unidades para disminuir los problemas psicológicos asociados al riesgo de fracaso académico. Además, hacer uso del modelo 
de cadenas de Markov para predecir el pronóstico de la intervención realizada en un grupo de estudiantes proporciona un valor añadido. El alcance de este trabajo y la intervención cognitivo conductual realizada son consideraciones importantes para los servicios y profesionales del ámbito universitario. Por lo tanto, los servicios universitarios de psicología pueden proporcionar beneficios sustanciales sobre el rendimiento y bienestar psicológico, al menos cuando se usa como un servicio adaptado a las necesidades del alumnado. En general, las universidades se enfrentan continuamente con la mejor manera de satisfacer las necesidades educativas de sus estudiantes en un contexto de recursos limitados. Este tipo de servicios ofrece muchas ventajas como una forma eficiente, asequible y eficaz de intervención y prevención, ya que precisamente ofrece intervenciones adaptadas a la sintomatología y necesidades del alumnado, y lo hace sin interferir en su proceso de enseñanza-aprendizaje.

La intervención psicológica realizada en este estudio llevó a la mejora del bienestar personal y académico del alumnado universitario que presentaban problemas o sintomatología asociada a trastornos psicológicos. Estos resultados son consistentes con las investigaciones previas y se suman a las evaluaciones que apoyan el uso de servicios de asesoramiento psicológicos y psicopedagógicos. Del mismo modo, estos hallazgos sugieren que el modelo de cadenas de Markov es útil cuando se quiere conocer un proceso que involucra riesgos que dependen del tiempo, y por ello puede ser un método complementario eficaz para analizar y predecir los resultados de una intervención.

No obstante, es necesario continuar investigando en esta línea con estudios que permitan analizar resultados con un grupo control, ampliando la muestra, generando protocolos estandarizados que posibiliten la generalización en su aplicación y concretando variables que intervengan directa o indirectamente en el bienestar y rendimiento académico de los estudiantes universitarios.

\section{Referencias}

Agudelo, D. M., Casadiegos, C. P., \& Sánchez, D. L. (2008). Características de ansiedad y depresión en estudiantes universitarios. International Journal of Psychological Research, 1(1), 34-39.

Aguilar, C., Alonso, M. J., Melgar, P., \& Molina, S. (2009). Violencia de género en el ámbito universitario. Medidas para su superación. Pedagogía Social, 16, 85-94.

Alcoba-González, J. (2000). Inax4: Un programa breve de intervencion en ansiedad. Estudio piloto. Indivisa: Boletín de Estudios e Investigación, 1, 197-221.

Alvarez, M. J., \& Garcia-Marques, L. (2011). Cognitive and contextual variables in sexual partner and relationship perception. Archives of Sexual Behavior, 40(2), 407-17. doi:http://doi.org/10.1007/s10508-011-9725-5

Arco, J. L., Fernandez, F. D., Heilborn, V. A., \& Lopez, S. (2005). Demographic, academic and psychological profile of students attending counseling services at the University of Granada (Spain). International Journal for the Advancement of Counselling, 27(1), 71-85. http://doi.org/10.1007/s10447-005-2249-8

Arco, J. L., López, S., Heilborn, V. A., \& Fernández, F. D. (2005). Terapia breve en estudiantes universitarios con problemas de rendimiento académico y ansiedad: Eficacia del modelo "La Cartuja." International Journal of Clinical and Health Psychology, 5(3), 589-608.

Arslan, G., Ayranci, U., Unsal, A., \& Arslantas, D. (2009). Prevalence of depression, its correlates among students, and its effect on health-related quality of life in a Turkish university. Upsala Journal of Medical Sciences, $\quad 114, \quad 170-177$. http://doi.org/10.1080/03009730903174339

Barkham, M., Margison, F., Leach, C., Lucock, M., Mellor-Clark, J., Evans, C., ... McGrath, G. (2001). Service profiling and outcomes benchmarking using the CORE-OM: Toward practice-based evidence in the psychological therapies. Journal of Consulting \& Clinical Psychology, 69, 184-196.

Bewick, B. M., Trusler, K., Mulhern, B., Barkham, M., \& Hill, A. J. (2008). The feasibility and effectiveness of a web-based personalised feedback and social norms alcohol intervention in UK university students: A randomised control trial. Addictive 
Behaviors,

33 ,

1192-1198.

doi:10.1016/j.addbeh.2008.05.002

Blanco, A., \& Díaz, D. (2005). El bienestar social: Su concepto y medición. Psicothema, 17(4), 582-589.

Botella, L. (2008). Resultado y proceso en psicoterapia cognitivo-constructivista integradora. Apuntes de PSicología, 26(2), 229-241.

Caparrós, B., Villar, E., Juan, J., \& Viñas, F. (2007). Symptom Check-List-90-R: Fiabilidad, datos normativos y estructura factorial en estudiantes universitarios. International Journal of Clinical and Health Psychology, 7(3), 781-794.

Casari, L. M., Isón, M., \& Gómez, B. (2018). Estilo personal del terapeuta: Estado actual (1998-2017). Revista Argentina de Clínica Psicológica, 27(3), 466-477. doi:10.24205/03276716.2018.1082

Chau, C., \& Saravia, J. C. (2016). Conductas de salud en estudiantes Universitarios limeños: Validación del CEVJU. Revista Iberoamericana de Diagnóstico y Evaluación - e Avaliação Psicológica, 41(1), 90-103.

Connell, J., Barkham, M., \& Mellor-Clark, J. (2007). CORE-OM mental health norms of students attending university counselling services benchmarked against an age-matched primary care sample. British Journal of Guidance \& Counselling, 35(1), 41-57. http://doi.org/10.1080/03069880601106781

Connell, J., Barkham, M., \& Mellor-Clark, J. (2008). The effectiveness of UK student counselling services: An analysis using the CORE System. British Journal of Guidance \& Counselling, $\quad 36(1), \quad 1-18$. http://doi.org/10.1080/03069880701715655

Connell, J., Barkham, M., Stiles, W. B., Twigg, E., Singleton, N., Evans, O., \& Miles, J. N. V. (2007). Distribution of CORE-OM scores in a general population, clinical cut-off points and comparison with the CIS-R. The British Journal of Psychiatry: The Journal of Mental Science, $\quad 190, \quad 69-74$. http://doi.org/10.1192/bjp.bp.105.017657

Dunne, C., \& Somerset, M. (2004) Health promotion in university: What do students want? Health Education, 104(6), 360-370. doi.org/10.1108/09654280410564132

Evans, C., Connell, J., Barkham, M., Margison,
F., McGrath, G., Mellor-Clark, J., \& Audin, K. (2002). Towards a standardised brief outcome measure: Psychometric properties and utility of the CORE--OM. The British Journal of Psychiatry, 180(1), 51-60. http://doi.org/10.1192/bjp.180.1.51

Gębka, B. (2013). Psychological determinants of university students' academic performance: An empirical study. Journal of Further and Higher Education, 38(6), 813-837. doi:10.1080/0309877X.2013.765945

Gutiérrez, J. A., Montoya, L. P., Toro, B. E., Briñón, M. A., Rosas, E., \& Salazar, L. E. (2010). Depresión en estudiantes universitarios y su asociación con el estrés académico. Revista CES Medicina, 24(1), 717.

Hallett, J., Maycock, B., Kypri, K., Howat, P., \& McManus, A. (2009). Development of a Webbased alcohol intervention for university students: Processes and challenges. Drug and Alcohol Review, 28, 31-39. doi:10.1111/j.1465-3362.2008.00008.x

Koutra, A., Katsiadrami, A., \& Diakogiannis, G. (2010). The effect of group psychological counselling in Greek university students' anxiety, depression, and self-esteem. European Journal of Psychotherapy \& Counselling, 12(2), 101-111. doi:10.1080/13642537.2010.482733

Lepp, A., Barkley, J. E., \& Karpinski, A. C. (2014). The relationship between cell phone use, academic performance, anxiety, and satisfaction with life in college students. Computers in Human Behavior, 31, 343-350. http://doi.org/10.1016/j.chb.2013.10.049

Marchena, E., Rapp, C., Hervías, F., Navarro, J. I., Alcalde, C., Aguilar, M., \& Menacho, I. (2009). El Servicio de Asesoramiento Psicológico (SAP) de la Universidad de Cádiz. Acción Psicológica, 6(1), 73-85.

Martín, V., Fernández, D., Ordóñez, C., Molina, A. J., \& Manuel, J. (2008). Valoración con tres métodos diferentes de la prevalencia de consumo de tabaco en estudiantes de primer curso de ciencias de la salud de la Universidad de León en 2006. Revista Española de Salud Pública, 85(2), 221-229.

Mehr, K. E., \& Daltry, R. (2016). Examining mental health differences between transfer 
and nontransfer university students seeking counseling services. Journal of College Student Psychotherapy, 30(2), 146-155. http://doi.org/10.1080/87568225.2016.114099 6

Micin, S., \& Bagladi, V. (2011). Salud mental en estudiantes universitarios: Incidencia de psicopatología y antecedentes de conducta suicida en población que acude a un servicio de salud estudiantil. Terapia Psicologica, 29(1), 53-64.

Minami, T., Davies, D. R., Tierney, S. C., Bettmann, J. E., McAward, S. M., Averill, L. A., ... Wampold, B. E. (2009). Preliminary evidence on the effectiveness of psychological treatments delivered at a university counseling center. Journal of Counseling Psychology, 56(2), 309-320. doi:10.1037/a0015398

Monti, F., Tonetti, L., \& Ricci Bitti, P. E. (2015). Short-term effectiveness of psychotherapy treatments delivered at a university counselling service. British Journal of Guidance \& Counselling, 1-9. http://doi.org/10.1080/03069885.2015.111923 3

Moreno-Yaguana, P. E., \& Sanchez-Garcia, M. F. (2018). Cuestionario de Necesidades de Orientación Universitaria. Revista Iberoamericana de Diagnóstico y Evaluación - e Avaliação Psicológica, 47(2), 95-109. https://doi.org/10.21865/RIDEP47.2.07

Murray, A. L., McKenzie, K., Murray, K. R., \& Richelieu, M. (2015). An analysis of the effectiveness of university counselling services. British Journal of Guidance \& Counselling, 44(1), 130-139. http://doi.org/10.1080/03069885.2015.1043621

Olani, A. (2009). Predicting first year University students' academic success. Electronic Journal of Research in Educational Psychology, 7, 1053-1072.

Ortís, L. C., Maymí, J. N., Feliu, J. C., Vidal, J. M. L., Romero, E. P., Bassets, M. P., ... Brosa, J. V. (2007). Exercise motivation in university community members: A behavioural intervention. Psicothema, 19(2), 250-255.

Palmieri, G., Evans, C., Hansen, V., Brancaleoni, G., Ferrari, S., Porcelli, P., ... Rigatelli, M. (2009). Validation of the Italian version of the
Clinical Outcomes in Routine Evaluation Outcome Measure (CORE-OM). Clinical Psychology \& Psychotherapy, 16(5), 444-9. http://doi.org/10.1002/cpp.646

Pullmann, H., \& Allik, J. (2008). Relations of academic and general self-esteem to school achievement. Personality and Individual Differences, 45(6), 559-564. doi:10.1016/j.paid.2008.06.017

Prat-Sala, M., \& Redford, P. (2010). The interplay between motivation, self-efficacy, and approaches to studying. The British Journal of Educational Psychology, 80(2), 283-305. doi:10.1348/000709909X480563

Raunic, A., \& Xenos, S. (2008). University counselling service utilisation by local and international students and user characteristics: A review. International Journal for the Advancement of Counselling, 30(4), 262-267. doi:10.1007/s10447-008-9062-0

Richardson, M., Abraham, C., \& Bond, R. (2012). Psychological correlates of university students' academic performance: A systematic review and meta-analysis. Psychological Bulletin, 138(2), 353-87. doi:10.1037/a0026838

Rosenthal, B., \& Wilson, W. C. (2008). Mental health services: Use and disparity among diverse college students. Journal of American College Health, 57(1), 61-67.

Sarokhani, D., Delpisheh, A., Veisani, Y., Sarokhani, M. T., Manesh, R. E., \& Sayehmiri, K. (2013). Prevalence of depression among university students: A systematic review and meta-analysis study. Depression Research and Treatment. doi:10.1155/2013/373857

Saúl, L. A., López-González, M. A., \& Bermejo, B. G. (2009). Revisión de los servicios de atención psicológica y psicopedagógica en las universidades españolas. Acción Psicológica, 6(1), 17-40.

Stringer, P., Crown, S., Lucas, C. J., \& Supramanium, S. (1977). Personality correlates of study difficulty and academic performance in university students. I. The Middlesex Hospital Questionnaire and Dynamic Personality Inventory. The British Journal of Medical Psychology, 50, 267-274.

Sursock, A., \& Smidt, H. (2010). Trends 2010: A 
decade of change in European Higher Education. European University Association (EUA). A free electronic version of this report is available through www.eua.be

Sursock, A. (2015). Trends 2015: Learning and Teaching in European Universities. European University Association (EUA) A free electronic version of this report is available through www.eua.be

Tirado, R., Aguaded, J. I., \& Marín, I. (2013). Incidencia de los hábitos de ocio y consumo de drogas sobre las medidas de prevención de la drogadicción en universitarios. Educación XX1: Revista de La Facultad de Educación, $16(2)$, 137-159. doi:10.5944/educxx1.16.2.2637

Vázquez, F. L., \& Blanco, V. (2008). Prevalence of DSM-IV major depression among spanish University students. Journal of American College Health, 57(2), 165-171.

Vladutiu, C. J., Martin, S. L., \& Macy, R. J. (2011). College - or university - based sexual assault prevention programs: A review of program outcomes, characteristics, and recommendations. Trauma, Violence \& Abuse, 12(2), 67-86. doi: $10.1177 / 1524838010390708$

Young, C. (2009). The CORE-OM intake norms of students attending a South African university counselling service: A comparison with UK counselling service data. British Journal of Guidance \& Counselling, 37, 473-483. http://doi.org/10.1080/03069880903161377 